\title{
Intercellular invasion of rice roots at the seedling stage by the rice false smut pathogen, Villosiclava virens
}

\author{
Kunlaya Prakobsub ${ }^{1}$ - Taketo Ashizawa ${ }^{2}$ (D)
}

Received: 22 December 2016 / Accepted: 17 May 2017 / Published online: 8 September 2017

(C) The Author(s) 2017. This article is an open access publication

\begin{abstract}
False smut is a serious disease affecting rice production worldwide. Initial infection of rice seedlings by Villosiclava virens was clarified using axenically cultured chlamydospores to inoculate rice roots. Chlamydospores were found on rice roots at 1 day post inoculation (dpi), and were germinating at 1-4 dpi. At 4 dpi, the infection germ tube had invaded the intercellular space between epidermal rice root cells. Between 5 and $11 \mathrm{dpi}$, branching and fusion-like structures were observed that may contribute to the establishment of the hyphal network on the root surface.
\end{abstract}

Keywords Ustilaginoidea $\cdot$ Clavicipitaceae $\cdot$ Infection process $\cdot$ Epidermal cells $\cdot$ Artificial inoculation

Rice false smut disease, caused by Villosiclava virens (anamorph Ustilaginoidea virens) (Takahashi 1896; Tanaka et al. 2008), reduces crop yield and rice quality. False smut balls (chlamydospore masses) are produced in grains on the panicle and fall onto the soil. The chlamydospores act as inocula on the next crop (Ashizawa et al. 2010). They also remain viable in the soil and can infect seedlings after planting. Although elongation of hyphae on rice roots at seedling stage (Schroud and TeBeest 2005), coleoptile infection by chlamydospores (Ikegami 1962) and fungal colonization of juvenile leaf sheaths attached to tiller buds (Tanaka et al.

Taketo Ashizawa

toketa@affrc.go.jp

1 Department of Plant Pathology, Faculty of Agriculture, Kasetsart University, 50 Ngam Wong Wan Rd, Ladyaow Chatuchak, Bangkok 10900, Thailand

2 National Agriculture and Food Research Organization, Central Region Agricultural Research Center, Tsukuba, Ibaraki 305-8666, Japan
2017) have been explored, little information is available detailing the initial infection process. To clarify the initial infection process of $V$. virens into rice roots at the seedling stage, we inoculated seedlings with axenically cultured chlamydospores of $V$. virens and followed germination and the route of invasion.

Seedlings of rice (Oryza sativa) variety Yumeaoba, which is susceptible to rice false smut disease, was used for inoculation. The seeds were soaked in a $0.5 \%(\mathrm{v} / \mathrm{v})$ solution of the fungicide Techleed C Flowable (ipconazole and copper; Kumiai Chemical Industry, Tokyo, Japan) at $26^{\circ} \mathrm{C}$ for $24 \mathrm{~h}$, and then kept in distilled water (DW) for 3 days at $26^{\circ} \mathrm{C}$. Germinated seeds were selected and soaked in $70 \%$ (v/v) ethanol for $5 \mathrm{~min}$ and rinsed twice in DW. The germinated seeds were cultured on Murashige and Skoog (MS) medium in plastic plates $(97 \times 140 \mathrm{~mm})$ at $25{ }^{\circ} \mathrm{C}$ under a $12 \mathrm{~h} / 12 \mathrm{~h}$ (light/dark) cycle for 7 days. Seedlings were selected and carefully removed from the agar, washed in DW, and then kept in a petri dish (9-cm diameter) until inoculation.

Dried barley seeds containing isolate U2003-1 of $V$. virens (Ashizawa et al. 2010) were cultured on brown rice medium (100 g brown rice and $100 \mathrm{~mL} \mathrm{DW})$ at $25^{\circ} \mathrm{C}$. At 1 month post-incubation (mpi), a mass of chlamydospores formed on the medium, which was covered with velvety yellow hyphae; the surface of the chlamydospore mass was gradually cracking open (Fig. 1). The chlamydospores slowly changed from yellow to deep orange until $5 \mathrm{mpi}$. In a preliminary inoculation test, chlamydospores at 1 to 4 mpi did not germinate on inoculated rice roots, and no infection hyphae were observed. For this reason, actively growing chlamydospores at 5 mpi were used for further study. For inoculum, 5-mpi chlamydospores were gently suspended in DW filtered through tissue paper and adjusted to $1 \times 10^{5}$ chlamydospores $/ \mathrm{mL}$. In addition, by transferring 


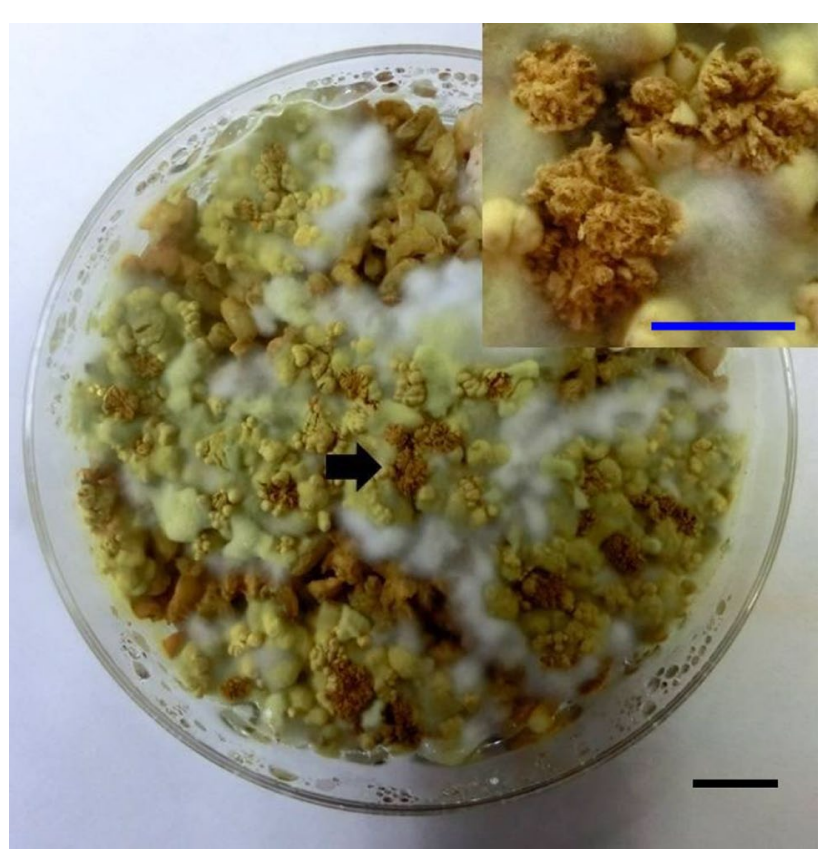

Fig. 1 Chlamydospores of Villosiclava virens isolate U2003-1 cultured on brown rice medium for 5 months. Bar $1 \mathrm{~cm}$. Upper right image shows a magnified chlamydospore mass (arrow). Blue bar $0.5 \mathrm{~cm}$

chlamydospore masses to new brown rice medium, a supply of inoculum was easily maintained.

For inoculation, we used the method of Schroud and TeBeest (2005) with minor modifications. Briefly, $10 \mathrm{~mL}$ of the chlamydospore suspension was added to a petri dish containing 7-day-old seedlings, then the dish was covered with parafilm. The inoculated seedlings were set vertically and kept at $25^{\circ} \mathrm{C}$ with a $12 \mathrm{~h} / 12 \mathrm{~h}$ (light/dark) cycle. Germinated chlamydospores were counted to calculate the percentage of viable chlamydospores on the rice roots; 30 chlamydospores were observed per replicate and three replicates per test ( 90 chlamydospores/test). For observations with a light microscope (BX43; Olympus, Tokyo, Japan), the inoculated rice roots were soaked in $70 \%(\mathrm{v} / \mathrm{v})$ ethanol for $5 \mathrm{~min}$ and then stained with $0.2 \%$ (w/v) trypan blue solution (Wako, Osaka, Japan) in a vacuum (-0.1 MPa) for $5 \mathrm{~min}$.

At 1-4 dpi, 22.0, 25.5, 29.1 and $36.6 \%$, respectively, of the chlamydospores had germinated on the inoculated roots. From 5 to $11 \mathrm{dpi}$, germination was constant at approximately $30 \%$. Our modified inoculation method results in successful deposition of $V$. virens chlamydospores on rice roots. At $1 \mathrm{dpi}$, the chlamydospores were consistently found on inoculated rice roots. The spiny nature of the chlamydospore cell wall surface (Kim and Park 2007) might facilitate their attachment to both roots and root hairs. The germination rate of chlamydospores on inoculated roots in our study was consistently higher than that previously reported (Schroud and TeBeest 2005). This might be caused by material differences between uniformly cultured chlamydospores and naturally harvested smut ball chlamydospores.

At $1 \mathrm{dpi}$, chlamydospores were distributed along the whole root (Fig. 2a) and the root hairs (Fig. 2b). Between 1 and 4 dpi, we observed four types of germ tubes produced after chlamydospore germination; a thick germ tube (Fig. 2c), a hook-like germ tube (Fig. 2d), an irregularly shaped germ tube (Fig. 2e), and a brownish, slightly swollen germ tube (Fig. 2f).

Penetration of the hyphae into the intercellular space between epidermal root cells (Fig. 2g, h) and hyphal elongation on the root surface (Fig. 2i) were observed at 4 dpi. In rare cases, at 6 and $8 \mathrm{dpi}$, brown, swollen hyphae had elongated (Fig. 2j), and irregular hyphae had branched (Fig. 2k) without penetrating root cells. Between 5 and 11 dpi, some elongated hyphae seemed to have fused, forming a thick fusion-like structure (Fig. $2 \mathrm{~m}$ ) and some had branched (Fig. 2n) on the root surface. Interestingly, at 9 dpi, hyphae had penetrated between epidermal root cells then elongated around the cell to grow back to the surface again (Fig. 21). Moreover, infection hyphae invaded intercellular spaces directly (Fig. 2o) during development of hyphae at low density (Fig. 2p). At 11 dpi, hyphae were abundant on the root surfaces (Fig. 2q). By contrast, floating chlamydospores in suspension had produced conidiophores (Fig. 2r) and conidium (Fig. 2s) at 2 dpi. In a rare case, a germ tube and conidium that formed on a chlamydospore were detected at 7 dpi (Fig. 2t).

At 1-4 dpi, we found thick germ tubes (Fig. 2c) and "hook-like" germ tubes (Fig. 2d). At 4 dpi, germ tubes had elongated and formed infection hyphae (Fig. $2 g$, h). On rare occasions, abnormal, irregularly shaped germ tubes (Fig. 2e) and slightly swollen germ tubes (Fig. 2f) were observed with light brown swollen hyphae at 6 dpi (Fig. 2j) or irregularly shaped hyphae (Fig. 2k) at 8 dpi. These germ tubes and hyphae were unable to penetrate and infect the root cells, potentially because of the physical difficulty they encountered in entering into the narrow space of the root cell. Additionally, conidia germinated on hair roots, but they did not infect the root hairs because no intercellular spaces are present for penetration. Interestingly, both conidia and germ tubes were produced in a chlamydospore showing a "two-faced" character (Fig. 2t) and may have occurred following removal of the chlamydospore from the root surface after germination on the root. From 5 to $11 \mathrm{dpi}$, infection hyphae invaded the intercellular space between epidermal cells (Fig. 21, o). This invasion type is consistent with that of an epitrophic fungus (Leonardi et al. 2006; Linskens 1976). By 11 dpi, a hyphal network substantially covered and grown within the inoculated roots (Fig. 2q). Hyphal fusion-like structures (Fig. 2m) and branching hyphae (Fig. 2n) may be involved in the development of this network, which may assist in the invasion of whole roots by $V$. virens. In our 

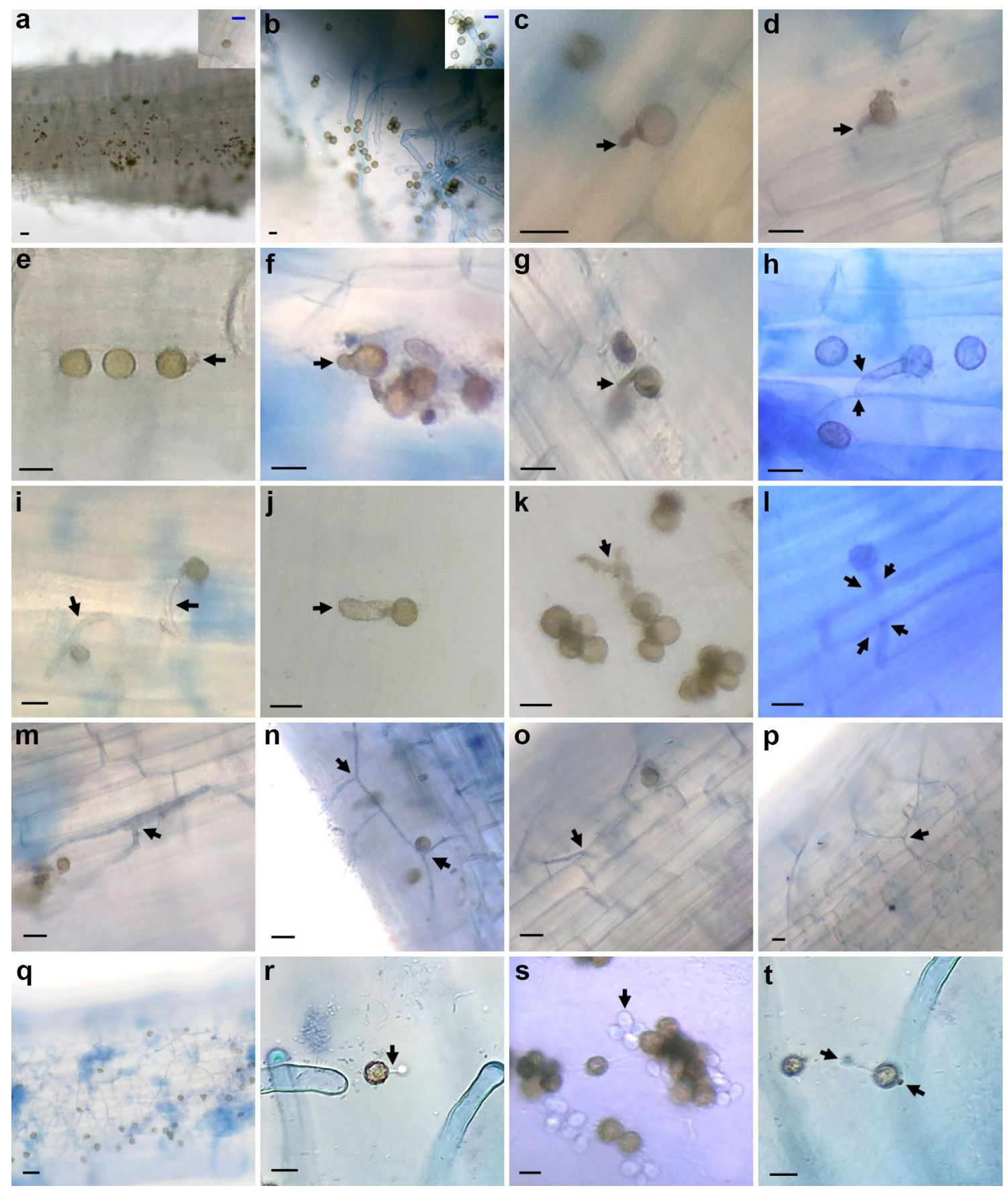

Fig. 2 Early development of Villosiclava virens from time of chlamydospore deposition and germination through germ tube elongation and infection hyphae invasion of the roots of rice seedlings. a Chlamydospores on rice root, 1 dpi. b Chlamydospores on root hairs (the upper right images in $\mathbf{a}, \mathbf{b}$ are magnifications showing the chlamydospores in greater detail). c Thick germ tube, 1 dpi. d Hook-like germ tube, 4 dpi. e Irregularly shaped germ tube, 3 dpi. f Brownish, slightly swollen germ tube, 2 dpi. g, h Germ tubes in intercellular spaces, 4 dpi. i Elongated germ tube, 4 dpi. j Brown, swollen germ tube, 6 dpi.

k Elongated, branched germ tube, 8 dpi. 1 Hypha on/in an epidermal root cell, as if sewn around the host cell, 9 dpi. m Hyphal fusionlike structure, 5 dpi. n Branching hyphae, 10 dpi. o Infection hyphae invading intercellular root cells, $11 \mathrm{dpi}$. p Hyphae at lower density, 11 dpi. q Hyphal network on and in the rice root, 11 dpi. r Chlamydospore producing a conidiophore and conidium, 2 dpi. s Conidia have formed on chlamydospores, 2 dpi. t Chlamydospore have produced conidium (left arrow) and a germ tube (right arrow), 7 dpi. Bars $\mathbf{a}, \mathbf{b}$, $\mathbf{p}, \mathbf{q} 10 \mu \mathrm{m} ; \mathbf{c}-\mathbf{o}, \mathbf{r}, \mathbf{s} 5 \mu \mathrm{m}$ 
experiment, the degree of staining differed among samples, probably as a result of differences in plant age and position of germination on rice roots.

In summary, we clarified the initial infection process of rice roots at the seedling stage by the rice false smut pathogen, $V$. virens. Our findings may contribute to the development of chemicals to suppress this invasion of rice roots. However, the progression of the rice false smut disease from the rice leaf bud at the seedling stage to infection of the rice floret on the panicle before the heading stage is still unknown and should be studied further to help clarify the entire invasion pathway.

Open Access This article is distributed under the terms of the Creative Commons Attribution 4.0 International License (http://creativecommons.org/licenses/by/4.0/), which permits unrestricted use, distribution, and reproduction in any medium, provided you give appropriate credit to the original author(s) and the source, provide a link to the Creative Commons license, and indicate if changes were made.

\section{References}

Ashizawa T, Takahashi M, Moriwaki J, Hirayae K (2010) Quantification of the rice false smut pathogen Ustilaginoidea virens from soil in Japan using real-time PCR. Eur J Plant Pathol 128:221-232
Ikegami H (1962) Study on the false smut of rice V. Seedling inoculation with the chlamydospores of the false smut fungus (in Japanese with English summary). Ann Phytopathol Soc Jpn 27:16-23

Kim KW, Park EW (2007) Ultrastructure of spined conidia and hyphae of the rice false smut fungus Ustilaginoidea virens. Micron 38:626-631

Leonardi PI, Miravalles AB, Faugeron S, Flores V, Beltrán J, Correa JA (2006) Diversity, phenomenology and epidemiology of epiphytism in farmed Gracilaria chilensis (Rhodophyta) in northern Chile. Eur J Phycol 41:247-257

Linskens HF (1976) Specific interactions in higher plants. In: Wood RKS, Graniti A (eds) Specificity in plant diseases. Plenum Press, New York, pp 311-326

Schroud P, TeBeest DO (2005) Germination and infection of rice roots by spores of Ustilaginoidea virens. AAES Res Ser 540:143-151

Takahashi Y (1896) On Ustilago virens Cooke and a new species of Tilletia parasitic on rice-plant. The Bot Mag (Tokyo) 10:16-20

Tanaka E, Ashizawa T, Sonoda R, Tanaka C (2008) Villosiclava virens gen. nov., comb. nov., teleomorph of Ustilaginoidea virens, the causal agent of rice false smut. Mycotaxon 106:491-501

Tanaka E, Kumagawa T, Ito N, Nakanishi A, Ohta Y, Suzuki E, Adachi N, Hamada A, Ashizawa T, Ohara T, Tsuda M (2017) Colonization of the vegetative stage of rice plants by the false smut fungus Villosiclava virens, as revealed by a combination of species-specific detection methods. Plant Pathol 66:56-66 